\title{
All fiber optical band-pass filter using macro-bending approach
}

\begin{abstract}
A new method for optical bandpass filters using macro bending approach is proposed. The proposed optical bandpass filter leverages on bending loss of higher order modes for shorter wavelengths. For longer wavelengths, the filter banks on fiber bending loss as the fundamental mode 'tail' is lost in the cladding. The combination of wavelength dependent loss at longer and shorter wavelength gives rise to the optical bandpass filter characteristics. We report both simulation and experimental results, which demonstrates agreement in the spectral response.
\end{abstract}

